\title{
The Optimization of Antioxidant Properties of Herbal Bathing Salts
}

\author{
K. ZaWADA*, K. Makarova, D. Kowalska And I. WaWer \\ Department of Physical Chemistry, Faculty of Pharmacy, The Medical University of Warsaw, \\ S. Banacha 1, 02-097 Warsaw, Poland
}

\begin{abstract}
The antioxidant properties 4 herbal infusions (of lavender flower, melissa leaf, verbena herb, and birch leaf), 2 salts (Dead Sea and Bochnia Mine salts) and 8 herbal salts (combination of herbal extract and salt) have been evaluated by 2,2-diphenyl-1-picrylhydrazyl-ESR (DPPH-ESR) test, ferric reducing antioxidant power and spin trap ESR using the Fenton reaction. The influence of herbal extracts and herbal salts on lipid membrane permeability has been studied with the spin probe ESR in the range of 293-323 K. The highest antioxidant activity was determined for melissa leaf preparations followed by birch leaf preparations in DPPH and ferric reducing antioxidant power assays whereas in the Fenton reaction the highest radical scavenging was observed for verbena herb and melissa leaf preparations. The lowest membrane permeability was observed in the presence of infusions of melissa and birch leaves, followed by corresponding herbal salts based on Bochnia Mine salt, and it was lower than in the reference system. On the other hand, lavender infusion increased the permeability of the model membrane, whereas the lavender salts decreased it. Therefore, the antioxidant activity of herbal infusions and salts towards skin could be inhibited by a lowered permeability. Thus, the combination of permeability studies with antioxidant studies in the presence of model lipids could be potentially used for optimization of other types of herbal bath salts.
\end{abstract}

DOI: 10.12693/APhysPolA.132.56

PACS/topics: 87.64.kh, 87.80.Lg

\section{Introduction}

Skin serves as a barrier to protect the body against water loss, solar radiation, pathogens etc., but it can lose its protective properties due to e.g. oxidative stress. A number of studies in cosmetic science demonstrated that the topical supplementation of the skin with various vitamins and other natural products with high antioxidant properties is beneficial for its functioning. Also, the benefits of the topical application of such minerals as magnesium and calcium was demonstrated in multiple balneotherapy studies, i.e. bathing in thermo-mineral waters or bath salts for home use [1, 2].

Salts obtained in different places have various courses of action, depending on their mineral composition. Thus, salt from the Dead Sea is one of the most studied skin cosmetic ingredients due to its unique mineral composition. It was reported that Dead Sea-derived salt could be used to treat various skin conditions, inflammatory skin disorders and arthritis [2-6]. Also it improves skin barrier function and enhances skin hydration. The main active compounds of Dead Sea salt are magnesium, calcium, sodium, potassium, zinc, strontium, sulfides, and bromides [7].

Another salt with noteworthy composition is Bochnia Mine salt. It is rich in calcium, magnesium, iodide and bromide [8]. Over 40 years old tradition of Bochnia Mine salt usage indicates that bathing with its addition helps in the prevention of hypothyroidism, dermatolog-

*corresponding author; e-mail: kzawada@wum.edu.pl ical, rheumatic and respiratory diseases, neuralgic ailments, and nervousness. It is postulated to have antiinflammatory, analgesic, and antiseptic properties [9].

The softening of the skin and pore dilation during a bath facilitates the absorption of other compounds, i.e. derived from herbal infusions. For example, melissa, verbena, birch or lavender infusions are traditionally used as bath additives for cosmetic and curative applications of various skin conditions. These plant materials have anti-inflammatory, wound healing and soothing properties [10-14]. These properties could be due to an antioxidant activity of the plants. The compounds responsible for the antioxidant properties of these plants are phenols [15], terpenes [16], derivatives of rosmarinic [17], and betulinic acid [18]. Thus, combination of salt and herbal extract could help to deliver these bioactive compounds to the skin.

In this work we studied the antioxidant properties of herbal bath salts prepared based on the salt from the Dead Sea and the Bochnia Mine combined with 4 herbal infusions, namely of birch (Betula verrucosa) leaf, lavender (Lavandula angustifolia Mill.) flower, melissa (Melisa officinalis) leaf and verbena (Verbena officinalis) herb. We used 2,2-diphenyl-1-picrylhydrazyl (DPPH) radical scavenging electron spin resonance (ESR), spin trapping ESR and ferric reducing antioxidant power (FRAP) assays to study the antioxidant properties of the selected salts with herbal extracts. Also, the spin probe ESR was used to test how the salts and the herbal salts influence the structure of the model membrane.

Our results showed that all studied plant materials have antioxidant activity. However, the presence of salts is essential for the permeation of the active compounds 
from melissa and birch leaves through the model membrane. Therefore, high antioxidant potential of the skin cosmetic revealed by commonly used DPPH or FRAP tests is not enough to extrapolate the results of positive health effect when the cosmetic is applied to skin. The spin probe studies of model membrane permeability could be a complementary method.

\section{Materials and methods}

\subsection{Materials}

Bochnia Mine salt was bought from Salco (Poland), and the Dead Sea salt from Meractiv (Dead Sea Jordan Trade Int. Poland). Birch leaf (Betulae folium), lavender flower (Lavandulae flos) and melissa leaf (Melisae folium) were purchased from Kawon (Poland), and the verbena herb (Verbena officinalis herba) from Dar Natury (Poland). DPPH radical, 2,4,6-tri(2-pyridyl)-striazine (TPTZ), PBN spin trap and DTBN (di-tertbutyl nitroxide) spin probe were purchased from SigmaAldrich (Germany), and ferrous sulfate $\left(\mathrm{FeSO}_{4} \cdot 7 \mathrm{H}_{2} \mathrm{O}\right)$, $\mathrm{NaH}_{2} \mathrm{PO}_{4}$ and $\mathrm{Na}_{2} \mathrm{HPO}_{4}$ from Avantor Performance Materials Poland (Poland). Hydrogen peroxide (30\%) and ethanol were purchased from Chempur. 1,2-dioleoyl-snglycero-3-phosphocholine (DOPC, 18:1PC) lipid solution in chloroform was obtained from Avanti Polar Lipids (USA). For all experiments the ultrapure water was used.

\subsection{Herbal salt preparation}

First, herbal infusions were prepared by water extraction of $5 \mathrm{~g}$ of dry herb with $100 \mathrm{ml}$ of water. The extraction was performed in temperature $333 \mathrm{~K}$ for $15 \mathrm{~min}$. Herbal salts were prepared by dissolving $1 \mathrm{~g}$ of salt in $2.5 \mathrm{ml}$ (Bochnia Mine salt) or $4.5 \mathrm{ml}$ (Dead Sea salt) of herbal infusion and recrystallization of salt at room temperature. Immediately before measurements the salt was dissolved in water in $0.2 \mathrm{~g} / \mathrm{ml}$ concentration.

\subsection{FRAP}

FRAP determination was performed according to Benzie and Strain [19].

\subsection{ESR}

The ESR measurements were performed on a MiniScope MS 200 spectrometer (Magnettech) using $50 \mathrm{\mu l}$ capillary tubes. Typical instrument settings were: sweep width $6.9 \mathrm{mT}$ (for spin trapping) and $7.2 \mathrm{mT}$ (for spin probe experiments), microwave power $10 \mathrm{~mW}$, modulation amplitude $0.05 \mathrm{mT}$, sweep time $20 \mathrm{~s}$. DPPH scavenging and spin trapping experiments were done at room temperature $(297 \pm 1 \mathrm{~K})$, and the spin probe experiments were performed with the temperature controller unit TC H03 (Magnettech).

\subsection{DPPH radical scavenging}

Ethanol solution of $0.5 \mathrm{~g} / \mathrm{l} \mathrm{DPPH}$ was used. The infusions and salts solutions were mixed with DPPH radical solution. The samples were incubated for $40 \mathrm{~min}$ in dark place at room temperature before ESR measurements.

\subsection{Spin trapping ESR}

Fenton reaction was used to generate free radicals. PBN solution was prepared in phosphate buffer, $\mathrm{pH}$ 7.5. To $20 \mu \mathrm{l}$ of $62.3 \mathrm{mM}$ PBN solution, $20 \mu \mathrm{l}$ of $20 \mathrm{mM} \mathrm{FeSO}_{4}$ solution, $20 \mu \mathrm{l}$ of $25 \mathrm{mM} \mathrm{H}_{2} \mathrm{O}_{2}, 20 \mu \mathrm{l}$ of $96 \%$ ethanol and $20 \mu \mathrm{l}$ of a sample (infusion or salt solution) were added. The sample was vortexed for $20 \mathrm{~s}$. The first measurement was performed after $110 \mathrm{~s}$ after addition of $\mathrm{H}_{2} \mathrm{O}_{2}$. The next 20 measurements were performed every $2.5 \mathrm{~min}$.

\subsection{Spin probe ESR}

$236 \mathrm{\mu l}$ of chloroform solutions of DOPC $(25 \mathrm{mg} / \mathrm{ml})$ were dried for $24 \mathrm{~h}$. Then $135 \mathrm{\mu l}$ of phosphate buffer $(\mathrm{pH} 7.5), 10 \mu \mathrm{l}$ of infusion or salt solution and $15 \mu \mathrm{l}$ of $7 \mathrm{mM}$ DTBN solution were added, the sample was vortexed for $2 \mathrm{~min}$ and then placed into ultrasound bath for $3 \mathrm{~min}$. This procedure results in formation dominantly of small unilamellar liposomes with a bimodal size distribution [20]. ESR spectra were registered in the temperature range $288-343 \mathrm{~K}$, with the temperature step $5 \mathrm{~K}$.

\subsection{ESR spectra simulations}

ESR spectra simulations were performed in Matlab using EasySpin toolbox [21], in order to obtain information about single components in obtained multicomponent ESR spectra. The spin adduct and spin probe spectra were simulated using "garlic" function. Hyperfine splitting, line broadening, correlation time, total intensity and ratio between components were optimized. The optimization of the parameters was performed using simplex method.

\section{Results and discussion}

\subsection{Antiradical and reducing properties}

The antioxidant activity of herbal infusions and herbal salts was studied with the most widely used tests for antiradical activity (DPPH test) and for reducing activity (FRAP), in order to determine whether the herbal salts exhibit any type of antioxidant properties.

The highest antiradical activity was obtained for melissa formulations, and the lowest for verbena (Fig. 1). The pure salts solutions were also studied, but did not show any radical scavenging properties. The infusions showed higher antiradical activity than the corresponding herbal salt solutions. However, the theoretical activity of salt solution, estimated on the basis of infusion:salt ratio, is 3 (Bochnia Mine salt) or 5 (Dead Sea salt) times higher than the activity of the infusion. It indicates the significant degradation of radical scavengers present in parent infusions during salt preparation.

The lower antiradical activity of verbena and lavender salts based on Bochnia Mine salt as compared with these based on Dead Sea salt could be due to the lower volume of infusion used for Bochnia Mine salt preparation. However, for melissa and birch the Bochnia Mine herbal salts have shown higher antiradical activity than Dead Sea salts. This discrepancy could be the result of different interactions between radical scavengers and metal ions present in salts. 


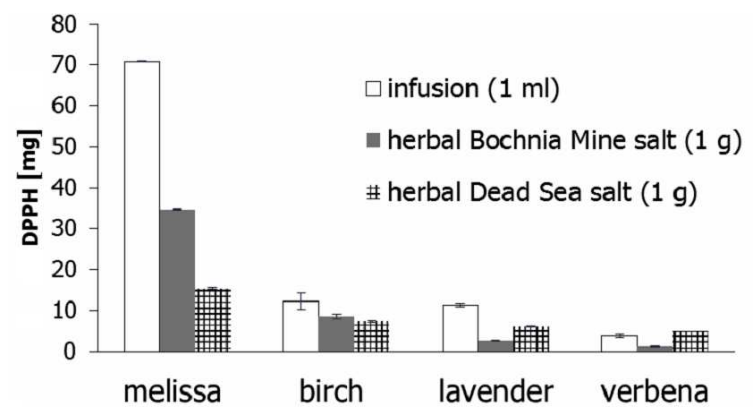

Fig. 1. DPPH radical scavenging of herbal infusions and herbal salts. White bars - infusion (per $1 \mathrm{ml}$ ), gray bars - herbal salts based on Bochnia Mine salt (per $1 \mathrm{~g}$ ), checked bars - herbal salts based on Dead Sea salt. Error bars represent the standard deviation of three replicates.

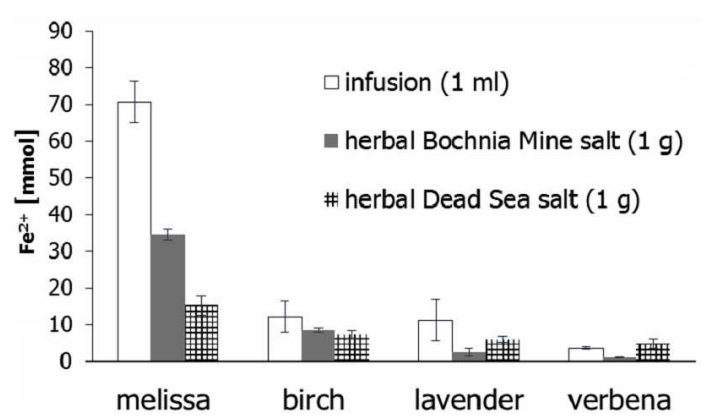

Fig. 2. Reducing properties of herbal infusions and herbal salts measured with FRAP test. White bars infusion (per $1 \mathrm{ml}$ ), gray bars - herbal salts based on Bochnia Mine salt (per $1 \mathrm{~g}$ ), checked bars - herbal salts based on Dead Sea salt. Error bars represent the standard deviation of three replicates.

As for the reducing activity, the highest value was again obtained for melissa preparations (Fig. 2), with the order of reducing strength: infusion $>$ Bochnia Mine salt $>$ Dead Sea salt. The same order was observed for preparations of birch. In case of lavender and verbena, the order of the reducing strength was: infusion $>$ Dead Sea salt $>$ Bochnia Mine salt. Still, the activity of Dead Sea salt was lower than the theoretical one, similarly to the DPPH-ESR test.

As the pure salts have shown no activity in FRAP test, the difference between the activity of Dead Sea and Bochnia Mine herbal salts cannot be caused directly by the high content of ferric ions in Dead Sea salt. Still, it seems that the compounds responsible for the reducing activity interact differently with the FRAP reagent in the presence of both salts, which is most probably due to the different mineral composition of both salts and possible side reactions.

As the next step, the activity of herbal infusions and salts towards short-lived radicals produced in Fenton reaction was studied, with the use of PBN spin trap. The ESR spectra simulation revealed that the same two com- ponents can be seen in all studied systems (example of simulation results is shown in Fig. 3). They were identified as $\mathrm{PBN} /{ }^{-} \mathrm{CHCH}_{3}(\mathrm{OH})$ and $\mathrm{PBN} / \cdot \mathrm{OH}$ radical adducts, on the basis of determined by spectral simulation hyperfine splitting constants values $\left(a_{\mathrm{N}}=1.59 \mathrm{mT}\right.$, $a_{\mathrm{H}}=0.23 \mathrm{mT}$ and $a_{\mathrm{N}}=1.48 \mathrm{mT}, a_{\mathrm{H}}=0.28 \mathrm{mT}$, respectively) $[22]$. The dominating radical adduct was $\mathrm{PBN} / \mathrm{CHCH}_{3}(\mathrm{OH})$ (about $95 \%$ of total intensity). In the case of the reference system the ratio between both components remained approximately constant during the whole monitored reaction time. When the herbal infusion or salt were present, they influenced almost exclusively the intensity and kinetics of formation of $\mathrm{PBN} / \mathrm{CHCH}_{3}(\mathrm{OH})$ adduct. The exceptions were the verbena infusion and verbena Bochnia Mine salt, where the intensity of $\mathrm{PBN} / \cdot \mathrm{OH}$ was decreased as well.

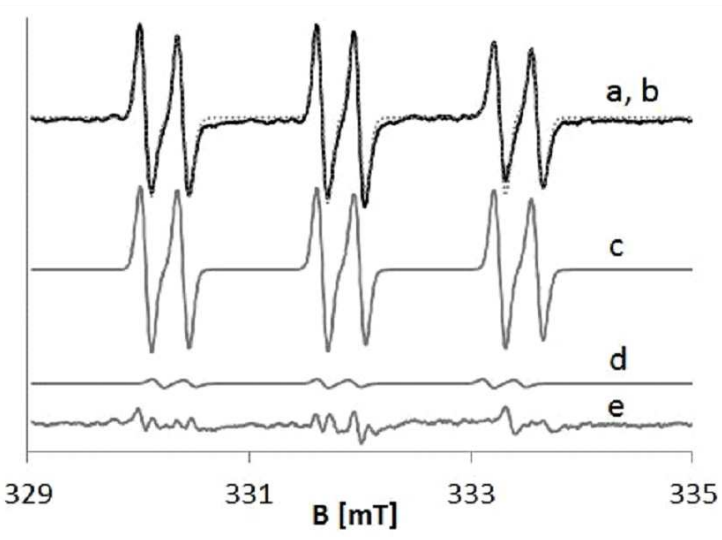

Fig. 3. ESR spectrum of Fenton reaction products in the presence of lavender infusion. a - experimental spectrum, b - simulated spectrum (dotted line), $\mathrm{c}-\mathrm{PBN} / \mathrm{CHCH}_{3}(\mathrm{OH})$ radical adduct component, $\mathrm{d}$ - $\mathrm{PBN} /{ }^{\circ} \mathrm{OH}$ radical adduct component, e - difference between experimental and simulated spectrum.

The relative total intensities after 5 and 20 min of Fenton reaction in studied systems are shown in Table I. The intensities were obtained by the double integration of ESR spectrum, and recalculated in relation to the intensity of a reference system signal at a given time. The reference system signal intensity was taken as $100 \%$. The nature and magnitude of observed effect depended both on the type of herb used for the preparation and on the type of parent salt. At first, after 5 min of reaction, in the presence of birch infusion and all herbal salts the intensity of ESR signal was lower than in the reference system. The lavender and melissa infusions had no influence on the signal intensity. However, after $20 \mathrm{~min}$ in the presence of birch and lavender infusions as well as of lavender and verbena Dead Sea salts the pro-oxidative effect was observed. In the presence of the rest of studied preparations the antioxidant effect, i.e. the decrease of radical adducts concentration, was observed. The shortterm (after 5 min of reaction) antioxidant effect was the strongest for verbena preparations, especially the Dead 
TABLE I

The relative intensities of ESR spectrum for Fenton reaction products in the presence of herbal infusions and herbal salts. DS - Dead Sea salt, BM - Bochnia Mine salt.

\begin{tabular}{l|c|c}
\hline \hline \multirow{2}{*}{} & \multicolumn{2}{|c}{ Relative total signal intensity } \\
\cline { 2 - 3 } & after 5 min & after 20 min \\
\hline reference & 100 & 100 \\
birch infusion & 78.8 & 120.0 \\
birch DS & 59.4 & 36.4 \\
birch BM & 81.0 & 38.5 \\
lavender infusion & 103.0 & 126.0 \\
lavender DS & 77.8 & 168.0 \\
lavender BM & 59.1 & 79.2 \\
melissa infusion & 101.2 & 45.4 \\
melissa DS & 64.0 & 38.9 \\
melissa BM & 79.0 & 78.9 \\
verbena infusion & 32.4 & 53.5 \\
verbena DS & 12.6 & 162.0 \\
verbena BM & 48.4 & 65.7
\end{tabular}

Sea salt, while after 20 min the best antioxidant properties towards short-living radicals were shown by birch herbal salts. The melissa preparations, which showed the best antiradical and reducing properties in DPPH-ESR and FRAP tests, exhibited moderate antioxidant activity towards radicals from Fenton reaction.

\subsection{Modification of model membrane permeability}

The potential pro-health activity of mineral and organic compounds in bath additives is connected with their ability to cross the cell membrane. It is known that salts can influence the fluidity of cell membrane thus changing the ease of penetration of other active substances into the skin cells. Therefore, the model lipid bilayer was used to estimate the potential influence of herbal salts on the fluidity of the membrane, as measured by the ability of a spin probe to penetrate it.

As a spin trap, DTBN has been chosen due to its hydrophilic properties and a small size, in order to better model the possibility of penetration of lipid bilayer by hydrophilic compounds such as these present in aqueous infusions of herbal material. Another reason was that even in lipid environment it gives ESR spectrum in the fast motion regime that can be analyzed with high precision.

As the first step, the influence of the herbal infusions and herbal salts on model lipid membrane (DOPC) was tested at room temperature $(295 \mathrm{~K})$. The ratio between spin probe in water and lipid phases was determined from ESR spectra simulations. The simulations were performed using $A_{\text {iso }}=1.70 \mathrm{mT}$, correlation time $3.1 \times 10^{-12} \mathrm{~s}$, broadening $0.03 \mathrm{mT}$ for the water phase, and $A_{\text {iso }}=1.57 \mathrm{mT}$, correlation time $2.9 \times 10^{-11} \mathrm{~s}$, broadening $0.05 \mathrm{mT}$ for the lipid phase. The difference in $g_{\text {iso }}$ for water and lipid phase was 0.00015 . The hyperfine splitting constants are consistent with the results of Windle [23]. The example of ESR spectrum of DTBN spin probe in liposome system together with simulation results is shown in Fig. 4. The ratios of spin probe in the water and the lipid phases at room temperature are shown in Table II. The percentage of spin probe in water phase increases in the presence of melissa and birch preparations, and this effect is the strongest for infusions. It should be noted that these preparations had the highest activity in DPPH and FRAP tests, and also showed noticeable activity in Fenton reaction test. However, the observed decrease of membrane fluidity especially in the presence of infusions indicates the potential problems with penetration of active compounds into the skin cells. Thus, only herbal salts were considered for further studies.

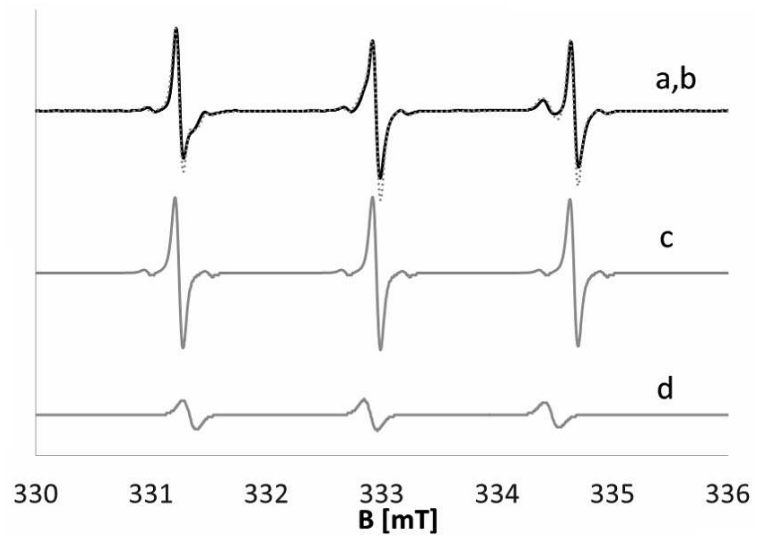

Fig. 4. ESR spectrum of DTBN spin probe in liposome system in the presence of a lavender salt based on Dead Sea salt. a - experimental spectrum, b - simulated spectrum (dotted line), c - DTBN signal in water phase, d - DTBN signal in lipid phase.

TABLE II

The percentage of spin probe (DTBN) in water phase, in DOPC liposomes system at room temperature.

\begin{tabular}{l|c}
\hline \hline & DTBN in water phase [\%] \\
\hline reference & 63 \\
birch infusion & 84 \\
birch DS & 62 \\
birch BM & 73 \\
lavender infusion & 64 \\
lavender DS & 65 \\
lavender BM & 64 \\
melissa infusion & 75 \\
melissa DS & 65 \\
melissa BM & 70 \\
verbena infusion & 61 \\
verbena DS & 62 \\
verbena BM & 60
\end{tabular}

Although the main phase transition $\left(L_{\beta}-L_{\alpha}\right.$; gelfluid) of lipid bilayer formed of DOPC lipids occurs at $256 \mathrm{~K}$, there has been observed an additional transition between 313 and $318 \mathrm{~K}$, connected with the bilayer thickness change and total volume increase (liquid-ordered to 
liquid-disordered transition) [24, 25]. Above the temperature of this transition, the fluidity of the membrane as well as its surface increases significantly [24]. Thus, at the temperature higher than $318 \mathrm{~K}$ the percentage of DTBN spin probe decreases in water and increases in lipid phase. In the presence of compounds which could affect the structure of the lipid bilayer this transition temperature could increase or decrease. It was shown that the presence of $\mathrm{Li}^{+}, \mathrm{Na}^{+}, \mathrm{K}^{+}$, and $\mathrm{Cs}^{+}$ions increased the membrane fluidity and decreased the $L_{\beta}-L_{\alpha}$ transition temperature [26]. At the same time, plant phenolics, which are the dominating active components of herbal aqueous infusions, can act similarly to cholesterol, i.e. increase the stiffness of the membrane and therefore increase the transition temperature [27, 28].

TABLE III

The phase transition temperature $T$ of DOPC liposomes and the change in spin probe DTBN percentage in water phase. DS - Dead Sea salt, BM — Bochnia Mine salt.

\begin{tabular}{l|c|c}
\hline \hline & $T[\mathrm{~K}]$ & DTBN decrease [\%] \\
\hline reference & $313-318$ & 6 \\
birch DS & $303-308$ & 4.5 \\
birch BM & $308-313$ & 6.5 \\
lavender DS & $293-298$ & 13.5 \\
lavender BM & $298-303$ & 5 \\
melissa DS & $308-313$ & 9 \\
melissa BM & $303-308$ & 6 \\
verbena DS & $298-303$ & 10 \\
verbena BM & $298-303$ & 6
\end{tabular}

Both lavender and verbena salts (Dead Sea and Bochnia Mine) decreased the transition temperature of DOPC lipid bilayer (Table III), although the Dead Sea salt effect was the stronger one. The effect of birch and melissa salts was less pronounced, but there was also a decrease in the transition temperature by $5-10 \mathrm{~K}$. The spectral parameters (hyperfine splitting, line broadening) have not changed observably after the transition. Still, the change of percentage of DTBN present in water phase indicated the occurrence of the transition. This change is shown in Table III. It should be noted that in the presence of all herbal salts the fluidity of membrane increased in the range of temperatures lower than the usual temperature of a bath (309-311 K). Thus, the combination of a mineral-rich salt and a herbal infusion could give an efficient method of application of bioactive substances.

\section{Conclusions}

Plants traditionally used for bathing revealed antioxidant activity. However, the observed trends in antioxidant activity of the herbal infusions and herbal salts depended on the kind of the test. It is most probably due to the different mechanisms of the reaction which each test is based on, and different affinity of various components of plants for reactants used in each assay. For example, melissa preparations showed the highest antioxidant activity in DPPH and FRAP tests, and moderate in Fenton reaction test. At the same time, birch and verbena had the highest antioxidant activity in Fenton reaction test, and showed low antioxidant activity in DPPH and FRAP tests. Thus, combination of several antioxidant tests with different underlying mechanisms of reaction should be used to assess the antioxidant activity of such plant extracts. Furthermore, the antioxidant activity in cosmetic applications could be influenced by the ability of active substances to cross the membrane. The presence of salt could modify this ability. Our results showed that verbena and lavender herbal salts improved the permeability of the model membrane in comparison with the infusions. Therefore it would seem that the ions present in salts influence the electrostatic interactions in the polar region of DOPC bilayers, reducing the density of the bilayer and counteracting the rigidifying effect of active compounds of plant, such as phenolics. Thus, combination of antioxidant and permeability studies gives the comprehensive information about the studied systems.

\section{References}

[1] T.G. Polefka, R.J. Bianchini, S. Shapiro, Int. J. Cosmet. Sci. 34, 416 (2012).

[2] E. Proksch, H.-P. Nissen, M. Bremgartner, C. Urquhart, Int. J. Dermatol. 44, 151 (2005).

[3] R. Schiffner, J. Schiffner-Rohe, G. Wölfl, M. Landthaler, A. Gläßl, Th. Walther, F. Hofstädter, W. Stolz, Br. J. Dermatol. 142, 740 (2000).

[4] J. Shani, S. Barak, D. Levi, M. Ram, E.R. Schachner, T. Schlesinger, H. Robberecht, R. Van Grieken, W.W. Avrach, Pharmacol. Res. Commun. 17, 501 (1985).

[5] M.E. Falagas, E. Zarkadoulia, P.I. Rafailidis, Int. J. Clin. Pract. 63, 1068 (2009).

[6] U. Katz, Y. Shoenfeld, V. Zakin, Y. Sherer, S. Sukenik, Semin. Arthritis Rheum. 42, 186 (2012).

[7] J. Wisniak, Indian J. Chem. Technol. 9, 79 (2002).

[8] K. Czajka, D. Sziwa, M. Drobnik, T. Latour, Pol. J. Balneol. 3, 176 (2006).

[9] Summary of product characteristics, Iodine-Bromide Bochenian salt (producer: Salco, in Polish).

[10] M. Deepak, S.S. Handa, Phytother. Res. 14, 463 (2000).

[11] M.I. Calvo, J. Ethnopharmacol. 107, 380 (2006).

[12] K. Ramanauskiene, A. Stelmakiene, D. Majiene, Evid. Based Complement. Alternat. Med. 2015, 635975 (2015).

[13] S. Sosa, G. Altinier, M. Politi, A. Braca, I. Morelli, R. Della Loggia, Phytomedicine 12, 271 (2005).

[14] S. Rastogi, M.M. Pandey, A.K.S. Rawat, J. Ethnopharmacol. 159, 62 (2015).

[15] C.-W. Lin, C.-W. Yu, S.-C. Wu, K.-H. Yih, J. Food Drug Anal. 17, 386 (2009).

[16] C. Lado, M. Then, I. Varga, E. Szóke, K. Szentmihályi, Z. Naturforsch. C 59, 354 (2004). 
[17] J. Lamaison, C. Petitjean-Freytet, A. Carnat, Ann. Pharm. Fr. 48, 103 (1990).

[18] P. Yogeeswari, D. Sriram, Curr. Med. Chem. 12, 657 (2005).

[19] I.F.F. Benzie, J.J. Strain, Anal. Biochem. 239, 70 (1996).

[20] N.-J. Cho, L.Y. Hwang, J.J.R. Solandt, C.W. Frank, Materials 6, 3294 (2013).

[21] S. Stoll, A. Schweiger, J. Magn. Reson. 178, 42 (2006).

[22] K. Makarova, K. Drązikowska, B. Suska, K. Zawada, I. Wawer, Nukleonika 60, 461 (2015).

[23] J.J. Windle, J. Magn. Reson. (1969) 45, 432 (1981).
[24] C. Dietrich, L.A. Bagatolli, Z.N. Volovyk, N.L. Thompson, M. Levi, K. Jacobson, E. Gratton, Biophys. J. 80, 1417 (2001).

[25] Z.V. Leonenko, E. Finot, H. Ma, T.E.S. Dahms, D.T. Cramb, Biophys. J. 86, 3783 (2004).

[26] R. Bartucci, L. Sportelli, Colloid Polym. Sci. 271, 262 (1993).

[27] D. Margina, D. Gradinaru, G. Manda, I. Neagoe, M. Ilie, Food Chem. Toxicol. 61, 86 (2013).

[28] H. Tsuchiya, M. Nagayama, T. Tanaka, M. Furusawa, M. Kashimata, H. Takeuchi, Biofactors 16, 45 (2002). 